\title{
On the tortuosity-connectivity of porous materials
}

\author{
M. Fenaux ${ }^{\mathrm{a}}$, E. Reyes ${ }^{\mathrm{a}}$, J.C. Gálvez ${ }^{\mathrm{a}, *}$, A. Moragues ${ }^{\mathrm{a}}$, J. Bernal ${ }^{\mathrm{a}}$ \\ ${ }^{a}$ Universidad Politécnica de Madrid \\ Escuela de Ingenieros de Caminos, Canales y Puertos \\ Madrid 28040, Spain
}

\begin{abstract}
In this work, the transport equations of ionic species in concrete are studied. First, the equations at the porescale are considered, which are then averaged over a representative elementary volume. The so obtained transport equations at the macroscopic scale are thoroughly examined and each term is interpreted. Furthermore, it is shown that the tortuosity-connectivity does not slow the average speed of the ionic species down. The transport equations in the representative elementary volume are then compared with the equations obtained in an equivalent pore. Lastly, comparing Darcy's law and the Hagen-Poiseuille equation in a cylindrical equivalent pore, the tortuosity-connectivity parameter is obtained for four different concretes. The proposed model provides very good results when compared with the experimentally obtained chloride profiles for two additional concretes.
\end{abstract}

Keywords: Tortuosity, Microscale-Macroscale, Averaging, Equivalent Pore, Ion Transport, Concrete

\section{Introduction}

The durability of concrete, which can be defined as its resistance to weathering action, chemical attacks and other degradation processes, is one of the most significant areas of research interest. Specifically, one of the most serious causes of durability problems affecting reinforcing steel is the introduction of chloride ions. The penetration of this aggressive ionic species into reinforced concrete induces steel corrosion and leads to premature deterioration of structures exposed to marine or de-icing salt environments $(2,3)$. As a result, most modern codes of practice have adopted strict maximum levels for chlorides permitted in concrete structures, among others the Spanish Standard of Structural Concrete EHE-08 (1). Therefore, studying the processes involved in transport of ionic species in concrete is of utmost benefit.

Accurate prediction of the behaviour of concrete in different environments has been a challenge until now. The means by which ions penetrate into concrete at a given exposure time are complex and depend on several variables of the material, particularly on the moisture of the material. Bertolini (4) pointed out that, among the different penetration mechanisms, four should be considered, namely diffusion, migration, absorption/capillary suction and permeation. An enormous variety of mathematical models has been proposed over the years with the main goal to explain and predict the penetration of ionic species into concrete $(5,6,7,8,9,10)$. The most simple models are generally based on the diffusion equation with an apparent diffusion coefficient which depends on the material as well as on the concentration of the ionic species $(11,12,13,14)$. The description of the various processes involved, requires the consideration of various transport mechanisms coupled with the effect of the interaction between the ion and the pore surface, which is very challenging.

The problem is further complicated when dealing with heterogeneous and porous materials, such as concrete. Indeed, the complex geometry of the porous network of cement-based materials is tortuous in nature. The geometry

\footnotetext{
*Corresponding author

Email address: jaime.galvez@upm.es (J.C. Gálvez)
} 
of the pathways of this materials for the penetration of aggressive agents is very complicated, even further due to its heterogeneity and particularly the higher porosity of the interface zone can facilitate the ingress of external aggressive agents and the development of deleterious chemical reactions. Bourdette et al. (? ) found a notably higher effective diffusion coefficient of chloride ions in the interface zone ( 6 to 12 times greater) than in the bulk cement paste. So, the detailed modelling of cement-based materials microstructure requires to consider different levels of approximation, which would greatly complicate the modelling. Seeking to more practical approaches, in previous works, a constant tortuosity factor has been adopted which assumes that all the effects arising from pore orientation, connectivity, size variation, etc., can be encompassed by a mean value valid for all pore sizes $(15,16,17)$. This is obviously a gross assumption since it depends on the implicit assumption that the effects of pore geometry and structure are the same for all pore sizes, even so, if the pore structure is characterized in detail, a sufficiently accurate aproach can be made. Ahmad (15) studied the relationship between permeability and tortuosity, based on the fact that both depend upon the pore structure and its interconnectivity.

This work focuses on the influence of the geometric complexity of the porous network on the different mass fluxes. First, the equations at the porescale are considered, which are then averaged over a representative elementary volume. This procedure shows the origin of the tortuosity-connectivity parameter used for modelling ionic transport in porous media. An explicit expression for the tortuosity-connectivity parameter is finally obtained by comparing Darcy's law and the Hagen-Poiseuille equation in a cylindrical equivalent pore. This expression can be written as a function of the pore radii, or more conveniently, as a function of the pore water content.

\section{General considerations}

In order to determine the differential equations which govern the transport problem at the macroscopic scale, the equations at the pore scale are integrated in a representative elementary volume (REV). The REV $\Omega$ is assumed to contain three phases, namely the liquid phase (the pore water), the gaseous phase (air) and the solid phase (concrete). Furthermore, it is assumed that the ionic species are transported solely through the liquid phase, and the solid phase is assumed to be inert. The REV can be seen as a whole of multiple elementary volumes. In this work, only homogeneous materials are considered, so that each elementary volume has the same properties. The characteristic length of the elementary volume is denoted as $l$, while the characteristic length of the REV is denoted as $L$. Furthermore, the size of the REV is chosen such that $\epsilon=l / L<<1$. A representation of one elementary volume, projected onto a 2D plane, is depicted in figure 1. The volume of the liquid phase is denoted as $\Omega_{l}$, while the boundaries between the phases $i$ and $j$ are denoted as $A_{i j}$. Finally, the unit vectors $\boldsymbol{n}_{i j}$ are normal to the $i-j$ interface pointing from the $i-$ phase to the $j-$ phase.

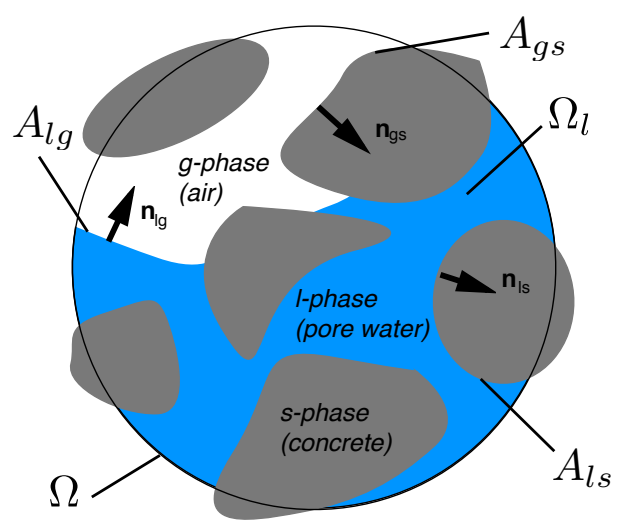

Figure 1: Elementary volume 
Throughout this work, the following notations are adopted:

$$
\begin{aligned}
& \langle\cdot\rangle_{\Omega}=\frac{1}{\Omega} \int_{\Omega} \cdot d V \\
& \langle\cdot\rangle_{\Omega_{l}}=\frac{1}{\Omega_{l}} \int_{\Omega_{l}} \cdot d V
\end{aligned}
$$

Note that equations 1-2 are related as follows:

$$
\langle\cdot\rangle_{\Omega}=\phi_{l}\langle\cdot\rangle_{\Omega_{l}}
$$

where $\phi_{l}=\Omega_{l} / \Omega$ is the pore water content.

Furthermore, let $u$ be a quantity related to the $l$-phase and consider the following decomposition (19):

$$
\begin{aligned}
u & =\langle u\rangle_{\Omega_{l}}+\tilde{u} \\
\langle\tilde{u}\rangle_{\Omega_{l}} & =0
\end{aligned}
$$

where $\tilde{u}$ is the deviation of $u$ with respect to the average $\langle u\rangle_{\Omega_{l}}$. From equation 5 , the following relations may be deduced:

$$
\begin{aligned}
\left\langle\langle u\rangle_{\Omega_{l}}\right\rangle_{\Omega_{l}} & =\langle u\rangle_{\Omega_{l}} \\
\tilde{\tilde{u}} & =\tilde{u}
\end{aligned}
$$

Equation 6 states that the average of the average value of $u$ in $\Omega_{l}$ is simply the average itself, while equation 7 states that the deviation of $\tilde{u}$ is equal to $\tilde{u}$.

Moreover, the following theorems are used throughout this work $(20,21,22)$ :

$$
\begin{array}{lrl}
\left\langle\frac{\partial u}{\partial t}\right\rangle_{\Omega} & =\frac{\partial}{\partial t}\langle u\rangle_{\Omega}-\frac{1}{\Omega} \int_{A_{l i}} u \boldsymbol{v}_{l i} \cdot \boldsymbol{n}_{l i} d A & \text { Reynolds } \\
\langle\nabla u\rangle_{\Omega}=\nabla\langle u\rangle_{\Omega}+\frac{1}{\Omega} \int_{A_{l i}} u \boldsymbol{n}_{l i} d A & \text { Averaging } \\
\langle\nabla . \boldsymbol{J}\rangle_{\Omega}=\nabla .\langle\boldsymbol{J}\rangle_{\Omega}+\frac{1}{\Omega} \int_{A_{l i}} \boldsymbol{J} \cdot \boldsymbol{n}_{l i} d A & \text { Averaging }
\end{array}
$$

where $\boldsymbol{J}$ is a vector quantity related to the $l$-phase, $i$ refers to the $g$-phase and the $s$-phase and $\boldsymbol{v}_{l i}$ is the velocity vector of the $l-i$ interface.

Equations 8-10 referred to the volume $\Omega_{l}$ read:

$$
\begin{aligned}
\left\langle\frac{\partial u}{\partial t}\right\rangle_{\Omega_{l}} & =\frac{1}{\phi_{l}} \frac{\partial}{\partial t}\left(\phi_{l}\langle u\rangle_{\Omega_{l}}\right)-\frac{1}{\Omega_{l}} \int_{A_{l i}} u v_{l i} \cdot \boldsymbol{n}_{l i} d A \\
\langle\nabla u\rangle_{\Omega_{l}} & =\frac{1}{\phi_{l}} \nabla\left(\phi_{l}\langle u\rangle_{\Omega_{l}}\right)+\frac{1}{\Omega_{l}} \int_{A_{l i}} u \boldsymbol{n}_{l i} d A \\
\langle\nabla . J\rangle_{\Omega_{l}} & =\frac{1}{\phi_{l}} \nabla \cdot\left(\phi_{l}\langle\boldsymbol{J}\rangle_{\Omega_{l}}\right)+\frac{1}{\Omega_{l}} \int_{A_{l i}} \boldsymbol{J} \cdot \boldsymbol{n}_{l i} d A
\end{aligned}
$$


Applying equations 8-9 to $u=1$, the following lemmas are obtained:

$$
\begin{aligned}
\frac{1}{\phi_{l}} \frac{\partial \phi_{l}}{\partial t} & =\frac{1}{\Omega_{l}} \int_{A_{l i}} \boldsymbol{v}_{l i} \cdot \boldsymbol{n}_{l i} d A \\
\frac{1}{\phi_{l}} \nabla \phi_{l} & =-\frac{1}{\Omega_{l}} \int_{A_{l i}} \boldsymbol{n}_{l i} d A
\end{aligned}
$$

Using equations 14-15, equations 11-12 can be rewritten as:

$$
\begin{aligned}
& \left\langle\frac{\partial u}{\partial t}\right\rangle_{\Omega_{l}}=\frac{\partial}{\partial t}\langle u\rangle_{\Omega_{l}}-\frac{1}{\Omega_{l}} \int_{A_{l i}} \tilde{u} \boldsymbol{v}_{l i} \cdot \boldsymbol{n}_{l i} d A \\
& \langle\nabla u\rangle_{\Omega_{l}}=\nabla\langle u\rangle_{\Omega_{l}}+\frac{1}{\Omega_{l}} \int_{A_{l i}} \tilde{u} \boldsymbol{n}_{l i} d A
\end{aligned}
$$

\section{The transport equations at the pore scale}

Denoting the concentration, mass flux and reaction terms of species $i$ by $u_{i}\left(\mathrm{~kg} / \mathrm{m}^{3}\right), \boldsymbol{J}^{i}\left(\mathrm{~kg} / \mathrm{m}^{2} / \mathrm{s}\right)$ and $r_{i}\left(\mathrm{~kg} / \mathrm{m}^{3} / \mathrm{s}\right)$, respectively, the transport equation at the pore scale is formulated as follows:

$$
\frac{\partial u_{i}}{\partial t}+\nabla . J^{i}=r_{i}
$$

where each term is expressed in units of mass per volume of pore water per second $\left(\mathrm{kg} / \mathrm{m}^{3} / \mathrm{s}\right)$.

The total mass flux is the sum of the diffusive mass flux $\boldsymbol{J}_{D}^{i}$, the advective mass flux $\boldsymbol{J}_{A}^{i}$, the mass flux related to migration $\boldsymbol{J}_{M}^{i}$ and the mass fluxes due to temperature gradients and chemical activity $\boldsymbol{J}_{T}^{i}(5)$ :

$$
\begin{aligned}
\boldsymbol{J}^{i} & =\boldsymbol{J}_{D}^{i}+\boldsymbol{J}_{A}^{i}+\boldsymbol{J}_{M}^{i}+\boldsymbol{J}_{T}^{i} \\
\boldsymbol{J}_{D}^{i} & =-D_{i} \nabla u_{i} \\
\boldsymbol{J}_{A}^{i} & =\boldsymbol{a} u_{i} \\
\boldsymbol{J}_{M}^{i} & =-\frac{z_{i} F}{R T} D_{i} u_{i} \nabla \Phi \\
\boldsymbol{J}_{T}^{i} & =-D_{i} u_{i} \gamma_{i}^{-1} \nabla \gamma_{i}-D_{i} u_{i} \ln \left(a_{i}\right) T^{-1} \nabla T
\end{aligned}
$$

where $D\left(\mathrm{~m}^{2} / \mathrm{s}\right)$ is the diffusion coefficient at infinite dilution and solely depends on temperature, $\boldsymbol{a}(\mathrm{m} / \mathrm{s})$ the advection velocity (i.e. the velocity of the pore water), $z$ the valence, $F(\mathrm{C} / \mathrm{mol})$ the Faraday constant, $R(\mathrm{~J} / \mathrm{mol} / \mathrm{K})$ the gas constant, $T(K)$ the temperature, $\Phi(V)$ the electric potential, $\gamma\left(\mathrm{m}^{3} / \mathrm{kg}\right)$ the activity coefficient and $a$ the chemical activity. An explicit expression for the advection velocity $\boldsymbol{a}$ will be given later.

Substitution of equations 19-23 into equation 18 yields:

$$
\begin{array}{r}
\frac{\partial u_{i}}{\partial t}+\nabla \cdot\left(\boldsymbol{a}_{i} u_{i}\right)=\nabla \cdot\left(D_{i} \nabla u_{i}+\frac{z_{i} F}{R T} D_{i} u_{i} \nabla \Phi+\right. \\
\left.D_{i} u_{i} \gamma_{i}^{-1} \nabla \gamma_{i}+D_{i} u_{i} \ln \left(a_{i}\right) T^{-1} \nabla T\right)+r_{i}
\end{array}
$$

\section{The transport equations at the macroscopic scale}

In order to obtain the equations at the macroscopic scale, the microscopic transport equation needs to be integrated in the REV. Thus, equation 24 is first integrated in the elementary volume $\Omega$ :

$$
\left\langle\frac{\partial u_{i}}{\partial t}\right\rangle_{\Omega}+\left\langle\nabla . J^{i}\right\rangle_{\Omega}=\left\langle r_{i}\right\rangle_{\Omega}
$$

In the following, each term will be treated separately. 


\subsection{The time derivative}

The average of the time derivative is obtained by applying Reynolds' transport theorem (equation 8 ) to the first term on the left-hand side of equation 24 :

$$
\begin{aligned}
\left\langle\frac{\partial u_{i}}{\partial t}\right\rangle_{\Omega}=\frac{\partial}{\partial t}\left\langle u_{i}\right\rangle_{\Omega} & -\frac{1}{\Omega} \int_{A_{l g}} u_{i} \boldsymbol{v}_{l g} \cdot \boldsymbol{n}_{l g} d A \\
& -\frac{1}{\Omega} \int_{A_{l s}} u_{i} \boldsymbol{v}_{l s} \cdot \boldsymbol{n}_{l s} d A
\end{aligned}
$$

It should be noted that the velocity of the $l-s$ interface is not necessarily equal to zero. Indeed, if the ionic species react to the cement matrix, the boundary between the $l$-phase and the $s$-phase may be deformed. However, for the sake of simplicity, it is assumed that this deformation does not alter significantly the transport of the ionic species, which simplifies equation 26 to:

$$
\left\langle\frac{\partial u_{i}}{\partial t}\right\rangle_{\Omega}=\frac{\partial}{\partial t}\left\langle u_{i}\right\rangle_{\Omega}-\frac{1}{\Omega} \int_{A_{l g}} u_{i} \boldsymbol{v}_{l g} \cdot \boldsymbol{n}_{l g} d A
$$

\subsection{The divergence of the mass fluxes}

The average of the divergence of the total mass flux is obtained by applying the averaging theorem (equation 10) to the second term of the left-hand side of equation 18 :

$$
\begin{aligned}
\left\langle\nabla . \boldsymbol{J}^{i}\right\rangle_{\Omega}=\nabla .\left\langle\boldsymbol{J}^{i}\right\rangle_{\Omega} & +\frac{1}{\Omega} \int_{A_{l g}} \boldsymbol{J}^{i} \cdot \boldsymbol{n}_{l g} d A \\
& +\frac{1}{\Omega} \int_{A_{l s}} \boldsymbol{J}^{i} \cdot \boldsymbol{n}_{l s} d A
\end{aligned}
$$

Since the ionic species are assumed to be transported solely through the $l$-phase, and assuming that the temperature is constant within the elementary volume, the following boundary conditions can be applied:

$$
\begin{aligned}
\boldsymbol{J}_{D}^{i} \cdot \boldsymbol{n}_{l g} & =0=\boldsymbol{J}_{D}^{i} \cdot \boldsymbol{n}_{l s} \\
\boldsymbol{J}_{A}^{i} \cdot \boldsymbol{n}_{l s} & =0 \\
\boldsymbol{J}_{M}^{i} \cdot \boldsymbol{n}_{l g} & =0=\boldsymbol{J}_{M}^{i} \cdot \boldsymbol{n}_{l s} \\
\boldsymbol{J}_{T}^{i} \cdot \boldsymbol{n}_{l g} & =0=\boldsymbol{J}_{T}^{i} \cdot \boldsymbol{n}_{l s}
\end{aligned}
$$

The assumption of a constant temperature within the elementary volume, which will be adopted in the rest of this paper, can be justified by the high thermal conductivity of concrete when compared with the slow penetration of the ionic species and the pore water (5). Moreover, it was found in (5) that temperature plays an important role in the transport of ionic species in concrete, while temperature gradients do not.

Now, the average of the divergence of the mass fluxes are obtained by means of equation 28 and the boundary conditions 29-32:

$$
\begin{aligned}
\left\langle\nabla . J_{D}^{i}\right\rangle_{\Omega} & =-D_{i}\left\langle\nabla \cdot\left(\nabla u_{i}\right)\right\rangle_{\Omega}=-D_{i} \nabla \cdot\left\langle\nabla u_{i}\right\rangle_{\Omega} \\
\left\langle\nabla . J_{A}^{i}\right\rangle_{\Omega} & =\left\langle\nabla .\left(\boldsymbol{a} u_{i}\right)\right\rangle_{\Omega}=\nabla \cdot\left\langle\boldsymbol{a} u_{i}\right\rangle_{\Omega}+\frac{1}{\Omega} \int_{A_{l g}} u_{i} \boldsymbol{a} \cdot \boldsymbol{n}_{l g} d A \\
\left\langle\nabla . \boldsymbol{J}_{M}^{i}\right\rangle_{\Omega} & =-\frac{z_{i} F}{R T} D_{i}\left\langle\nabla \cdot\left(u_{i} \nabla \Phi\right)\right\rangle_{\Omega}=-\frac{z_{i} F}{R T} D_{i} \nabla \cdot\left\langle u_{i} \nabla \Phi\right\rangle_{\Omega} \\
\left\langle\nabla . J_{T}^{i}\right\rangle_{\Omega} & =-D_{i}\left\langle\nabla \cdot\left(u_{i} \gamma_{i}^{-1} \nabla \gamma_{i}+u_{i} \ln \left(a_{i}\right) T^{-1} \nabla T\right)\right\rangle_{\Omega} \\
& =-D_{i} \nabla \cdot\left\langle u_{i} \gamma_{i}^{-1} \nabla \gamma_{i}+u_{i} \ln \left(a_{i}\right) T^{-1} \nabla T\right\rangle_{\Omega}
\end{aligned}
$$




\subsection{The transport equations at the macroscopic scale}

Substituting equation 27 and equations 33-36 into equation 25, the transport equations at the macroscopic scale are obtained:

$$
\begin{aligned}
\frac{\partial}{\partial t}\left\langle u_{i}\right\rangle_{\Omega} & +\nabla \cdot\left\langle\boldsymbol{a} u_{i}\right\rangle_{\Omega}+\frac{1}{\Omega} \int_{A_{l g}} u_{i}\left(\boldsymbol{a}-\boldsymbol{v}_{l g}\right) \cdot \boldsymbol{n}_{l g} d A \\
& =D_{i} \nabla \cdot\left\langle\nabla u_{i}\right\rangle_{\Omega}+\frac{z_{i} F}{R T} D_{i} \nabla \cdot\left\langle u_{i} \nabla \Phi\right\rangle_{\Omega} \\
& +D_{i} \nabla \cdot\left\langle u_{i} \gamma_{i}^{-1} \nabla \gamma_{i}+u_{i} \ln \left(a_{i}\right) T^{-1} \nabla T\right\rangle_{\Omega}+\left\langle r_{i}\right\rangle_{\Omega}
\end{aligned}
$$

The velocity $\boldsymbol{v}_{l g}$ represents the total velocity of the $l-g$ interface, while $\boldsymbol{a}$ at the boundary is the velocity of the interface due to the movement of the $l$-phase. In other words, unlike $\boldsymbol{a}, \boldsymbol{v}_{l g}$ accounts for the velocity of the interface due to the evaporation and condensation of the pore water. The velocity $\boldsymbol{v}_{l g}$ can thus be interpreted as the sum of the velocity at the $l-g$ interface $\left.\boldsymbol{a}\right|_{l g}$ and the velocity of the boundary due to evaporation and condensation $\boldsymbol{v}_{l g, v a p}$ :

$$
v_{l g}=\left.a\right|_{l g}+v_{l g, v a p}
$$

Combining equations 14 and 38, the variations of the pore water content with time can be expressed as follows:

$$
\frac{\partial \phi_{l}}{\partial t}=\frac{1}{\Omega} \int_{A_{l g}} \boldsymbol{a} \cdot \boldsymbol{n}_{l g} d A+\frac{1}{\Omega} \int_{A_{l g}} \boldsymbol{v}_{l g, v a p} \cdot \boldsymbol{n}_{l g} d A
$$

where the second surface integral of equation 39 represents the change of $\phi_{l}$ with time due to evaporation and condensation of the pore water. Mainguy et al. (23) showed that when concrete is subjected to drying processes the pore water is evaporated at the surface of the material, and the evaporation within the material can be ignored. Therefore, the effects of evaporation and condensation within the elementary volume are neglected, so that $\boldsymbol{v}_{l g, v a p}=\mathbf{0}$.

Equation 37 can now be rewritten as:

$$
\begin{gathered}
\frac{\partial}{\partial t}\left\langle u_{i}\right\rangle_{\Omega}+\nabla \cdot\left\langle\boldsymbol{a} u_{i}\right\rangle_{\Omega}=D_{i} \nabla \cdot\left\langle\nabla u_{i}\right\rangle_{\Omega}+\frac{z_{i} F}{R T} D_{i} \nabla \cdot\left\langle u_{i} \nabla \Phi\right\rangle_{\Omega}+ \\
D_{i} \nabla \cdot\left\langle u_{i} \gamma_{i}^{-1} \nabla \gamma_{i}+u_{i} \ln \left(a_{i}\right) T^{-1} \nabla T\right\rangle_{\Omega}+\left\langle r_{i}\right\rangle_{\Omega}
\end{gathered}
$$

or with respect to volume $\Omega_{l}$ :

$$
\begin{aligned}
& \frac{\partial}{\partial t}\left(\phi_{l}\left\langle u_{i}\right\rangle_{\Omega_{l}}\right)+\nabla \cdot\left(\phi_{l}\left\langle\boldsymbol{a} u_{i}\right\rangle_{\Omega}\right) \\
& =D_{i} \nabla \cdot\left(\phi_{l}\left\langle\nabla u_{i}\right\rangle_{\Omega_{l}}\right)+\frac{z_{i} F}{R T} D_{i} \nabla \cdot\left(\phi_{l}\left\langle u_{i} \nabla \Phi\right\rangle_{\Omega_{l}}\right) \\
& +D_{i} \nabla \cdot\left(\phi_{l}\left\langle u_{i} \gamma_{i}^{-1} \nabla \gamma_{i}+u_{i} \ln \left(a_{i}\right) T^{-1} \nabla T\right\rangle_{\Omega_{l}}\right)+\phi_{l}\left\langle r_{i}\right\rangle_{\Omega_{l}}
\end{aligned}
$$

\subsection{The mass fluxes}

Explicit expressions for the mass fluxes are given in equations 20-23, except for the advective mass flux, which depends on the advection velocity $\boldsymbol{a}$. The advection velocity is not known a priori and depends strongly on the pore water content $\phi_{l}$. In this work, $\boldsymbol{a}$ is modelled by means of Darcy's law, based on research work (5):

$$
\boldsymbol{a}=\frac{k_{l}}{v_{l}} \nabla p_{c}
$$

where $k_{l}\left(\mathrm{~m}^{2}\right)$ is the permeability of the porous medium, $v_{l}(\mathrm{~Pa} \cdot s)$ is the dynamic viscosity of the pore water, and $p_{c}(\mathrm{~Pa})$ is the capillary pressure. The permeability of the porous medium relative to the pore water depends on the pore water content, the dynamic viscosity of the pore water depends on the concentration of the ionic species and on temperature, and the capillary pressure depends on the pore water content, temperature and porosity (5). As the 
porosity can change due to chemical reactions of the ionic species with the cement matrix, and those reactions depend directly on the concentration of the ions, the capillary pressure may be expressed as a function of the concentration, rather than the porosity.

The advective mass flux (21) at the pore scale now reads:

$$
J_{A}^{i}=u_{i} \frac{k_{l}}{v_{l}} \nabla p_{c}
$$

The averages of the mass fluxes are obtained by applying the average theorem (equation 9) and the decomposition, as shown in equation 4 to the fluxes defined in equations 20, 22-23 and 43:

$$
\begin{aligned}
\left\langle\boldsymbol{J}_{D}^{i}\right\rangle_{\Omega}= & -D_{i}\left(\nabla\left\langle u_{i}\right\rangle_{\Omega}+\frac{1}{\Omega} \int_{A_{l j}} u_{i} \boldsymbol{n}_{l j} d A\right) \\
\left\langle\boldsymbol{J}_{A}^{i}\right\rangle_{\Omega}= & \left\langle u_{i}\right\rangle_{\Omega} \phi_{l}^{-1} \frac{k_{l}}{v_{l}}\left(\nabla\left\langle p_{c}\right\rangle_{\Omega}+\frac{1}{\Omega} \int_{A_{l j}} p_{c} \boldsymbol{n}_{l j} d A\right) \\
& +\frac{k_{l}}{v_{l}}\left\langle\tilde{u}_{i} \nabla p_{c}\right\rangle_{\Omega} \\
\left\langle\boldsymbol{J}_{M}^{i}\right\rangle_{\Omega}= & -\frac{z_{i} F}{R T} D_{i}\langle u\rangle_{\Omega_{l}}\left(\nabla\langle\Phi\rangle_{\Omega}+\frac{1}{\Omega} \int_{A_{l j}} \Phi \boldsymbol{n}_{l j} d A\right) \\
& -\frac{z_{i} F}{R T} D_{i}\langle\tilde{u} \nabla \Phi\rangle_{\Omega} \\
\left\langle\boldsymbol{J}_{T}^{i}\right\rangle_{\Omega}= & -D_{i}\left\langle u_{i}\right\rangle_{\Omega_{l}}\left(\nabla\left\langle\ln \gamma_{i}\right\rangle_{\Omega}+\left\langle\ln a_{i}\right\rangle_{\Omega_{l}} \nabla\langle\ln T\rangle_{\Omega}\right. \\
+ & \left.\frac{1}{\Omega} \int_{A_{l j}}\left(\ln \gamma_{i}+\left\langle\ln a_{i}\right\rangle_{\Omega_{l}} \ln T\right) \boldsymbol{n}_{l j} d A\right) \\
& -D_{i}\left\langle\tilde{u}_{i} \nabla \ln \gamma_{i}+\left(\tilde{u}_{i} \ln a_{i}+\left\langle u_{i}\right\rangle_{\Omega_{l}} \ln a_{i}\right) \nabla \ln T\right\rangle_{\Omega}
\end{aligned}
$$

where the subscript $j$ refers to the $g-$ and $s$-phases.

Note that, in equation 44 , there is no need to calculate the average of the diffusion coefficient. Indeed, $D$ solely depends on temperature which is assumed to be constant in the elementary volume. Furthermore, it should be noted that equation 45 was obtained by assuming that the deviations of $u_{i}$ within the elementary volume are sufficiently small so that the dynamic viscosity $v_{l}$ can be assumed constant in the elementary volume. This approximation can be expressed as follows:

$$
v_{l}\left(u_{i}, T\right)=v_{l}\left(\left\langle u_{i}\right\rangle_{\Omega_{l}}+\tilde{u}, T\right) \simeq v_{l}\left(\left\langle u_{i}\right\rangle_{\Omega_{l}}, T\right)
$$

Substitution of the expressions 44-48 into equation 40 yields the final transport equation in the elementary volume:

$$
\begin{aligned}
& \frac{\partial}{\partial t}\left\langle u_{i}\right\rangle_{\Omega}+\nabla \cdot\left(\left\langle u_{i}\right\rangle_{\Omega} \phi_{l}^{-1} \frac{k_{l}}{v_{l}}\left(\nabla\left\langle p_{c}\right\rangle_{\Omega}+\frac{1}{\Omega} \int_{A_{l j}} p_{c} \boldsymbol{n}_{l j} d A\right)\right) \\
& =D_{i} \nabla \cdot\left(\nabla\left\langle u_{i}\right\rangle_{\Omega}+\frac{1}{\Omega} \int_{A_{l i}} u_{i} \boldsymbol{n}_{l j} d A\right) \\
& +\frac{z_{i} F}{R} \nabla \cdot\left(D_{i} T^{-1}\left\langle u_{i}\right\rangle_{\Omega_{l}}\left[\nabla\langle\Phi\rangle_{\Omega}+\frac{1}{\Omega} \int_{A_{l j}} \Phi \boldsymbol{n}_{l j} d A\right]\right)
\end{aligned}
$$




$$
\begin{aligned}
& +\nabla \cdot\left(D _ { i } \langle u _ { i } \rangle _ { \Omega _ { l } } \left[\nabla\left\langle\ln \gamma_{i}\right\rangle_{\Omega}+\left\langle\ln a_{i}\right\rangle_{\Omega_{l}} \nabla\langle\ln T\rangle_{\Omega}\right.\right. \\
& \left.\left.+\frac{1}{\Omega} \int_{A_{l j}}\left(\ln \gamma_{i}+\left\langle\ln a_{i}\right\rangle_{\Omega_{l}} \ln T\right) \boldsymbol{n}_{l j} d A\right]\right) \\
& +\frac{z_{i} F}{R} \nabla \cdot\left(D_{i} T^{-1}\left\langle\tilde{u}_{i} \nabla \Phi\right\rangle_{\Omega}\right)+\nabla \cdot\left(D_{i}\left\langle\tilde{u}_{i} \nabla \ln \gamma_{i}\right\rangle_{\Omega}\right) \\
& +\nabla \cdot\left(D_{i}\left\langle\left[\tilde{u}_{i} \ln a_{i}+\left\langle u_{i}\right\rangle_{\Omega_{l}} \widetilde{\ln a_{i}}\right] \nabla \ln T\right\rangle_{\Omega}\right) \\
& -\nabla \cdot\left(\frac{k_{l}}{v_{l}}\left\langle\tilde{u}_{i} \nabla p_{c}\right\rangle_{\Omega}\right)+\left\langle r_{i}\right\rangle_{\Omega}
\end{aligned}
$$

\subsection{The dispersion terms}

In this subsection, it is shown that the dispersion terms of equation 50 can be ignored in the REV. To that end, consider a quantity $m$ related to the $l$-phase. According to equation $4, m$ can be decomposed as:

$$
m=\langle m\rangle_{\Omega_{l}}+\tilde{m}
$$

Since the microscopic quantity $m$ may vary significantly over a distance equal to the characteristic length $l$, the magnitude of the deviation of $m$ with respect to the average $\langle m\rangle_{\Omega_{l}}$ can be estimated as follows:

$$
\|\tilde{m}\| \simeq l\|\nabla m\|
$$

Furthermore, the magnitude of a quantity at the macroscale only varies significantly over distances larger than $L$, which can be expressed as:

$$
\left\|\nabla \cdot\langle\cdot\rangle_{\Omega}\right\| \simeq L^{-1}\left\|d\langle\cdot\rangle_{\Omega}\right\|
$$

Applying those approximations to equation 50, and noting that the diffusion coefficient can change within the REV due to temperature variations, the final transport equation in the REV is obtained:

$$
\begin{aligned}
& \frac{\partial}{\partial t}\left\langle u_{i}\right\rangle_{\hat{\Omega}}+\nabla \cdot\left(\left\langle u_{i}\right\rangle_{\hat{\Omega}} \phi_{l}^{-1} \frac{k_{l}}{v_{l}}\left[\nabla\left\langle p_{c}\right\rangle_{\hat{\Omega}}+\frac{1}{\hat{\Omega}} \int_{\hat{A}_{l j}} p_{c} \boldsymbol{n}_{l j} d A\right]\right) \\
& =\nabla \cdot\left(D_{i}\left[\nabla\left\langle u_{i}\right\rangle_{\hat{\Omega}}+\frac{1}{\hat{\Omega}} \int_{\hat{A}_{l j}} u_{i} \boldsymbol{n}_{l j} d A\right]\right) \\
& +\frac{z_{i} F}{R} \nabla \cdot\left(D_{i} T^{-1}\left\langle u_{i}\right\rangle_{\hat{\Omega}}\left[\nabla\langle\Phi\rangle_{\hat{\Omega}}+\frac{1}{\hat{\Omega}} \int_{\hat{A}_{l j}} \Phi \boldsymbol{n}_{l j} d A\right]\right) \\
& +\nabla \cdot\left(D _ { i } \langle u _ { i } \rangle _ { \hat { \Omega } } \left[\nabla\left\langle\ln \gamma_{i}\right\rangle_{\hat{\Omega}}+\left\langle\ln a_{i}\right\rangle_{\hat{\Omega}_{l}} \nabla\langle\ln T\rangle_{\hat{\Omega}}\right.\right. \\
& \left.\left.+\frac{1}{\hat{\Omega}} \int_{\hat{A}_{l j}}\left(\ln \gamma_{i}+\left\langle\ln a_{i}\right\rangle_{\hat{\Omega}_{l}} \ln T\right) \boldsymbol{n}_{l j} d A\right]\right)+\left\langle r_{i}\right\rangle_{\hat{\Omega}}
\end{aligned}
$$

where $\hat{\Omega}, \hat{\Omega}_{l}$ and $\hat{A}$ are related to the REV.

\subsection{The surface integrals}

From equation 54, it may be observed that all the mass fluxes in the REV have the form:

$$
\left\langle\boldsymbol{J}^{i}\right\rangle_{\hat{\Omega}}=f\left(\nabla\langle\cdot\rangle_{\hat{\Omega}}+\frac{1}{\hat{\Omega}} \int_{\hat{A}_{l j}} \cdot \boldsymbol{n}_{l j} d A\right)
$$

where $\boldsymbol{J}^{i}$ is a mass flux of species $i$ and $f$ is a scalar. 
In order to interprete the terms on the right-hand side of equation 55, consider an elementary volume with only one straight cylindrical pore. The REV can then be thought of as a volume with a single cylindrical pore. In such case, the second term on the right-hand side of equation 55 is zero. Therefore, the first term can be interpreted as the mass flux through the cylindrical pore. The second term corrects the mass flux by accounting for the complex geometry of the porous medium as shown below.

\subsubsection{The surface integrals at the pore scale}

The second term on the right-hand side of equation 55 is often associated with the tortuosity-connectivity of the porous medium. Generally, the more tortuous the porous material is, the slower is the penetration of the ionic species. However, it should be noted that the term which accounts for the geometry of the porous network does not slow down the speed of the ionic species. To illustrate this, the simple diffusion equation at the pore scale for some ionic species $i$ is considered:

$$
\frac{\partial u_{i}}{\partial t}=D_{i} \Delta u_{i}
$$

where $\Delta$ is the Laplace operator.

Two kinds of pores are analysed, namely a straight cylindrical pore and a tortuous pore. The dimensions of the pores were chosen such that the length and volume of each pore were equal.

A concentration of $u_{i}=1 \mathrm{~kg} / \mathrm{m}^{3}$ was imposed on the left boundary of the pores. The remaining boundaries are taken to be the $l-s$ interface, where a zero mass flux is imposed. The value of the diffusion coefficient was chosen such that:

$$
\frac{t_{\text {sim }} D_{i}}{d^{2}}=1
$$

where $t_{\text {sim }}$ refers to the total simulation time, and $d$ the length of the pore.

Equation 56 was solved by means of the finite element method. The results at time $t=0.38 t_{\text {sim }}$ are shown in figures 2-3. It may be observed that the ionic species reach the right boundary faster in the tortuous pore than in the cylindrical pore. This shows that tortuosity-connectivity increases the average speed of the ions.

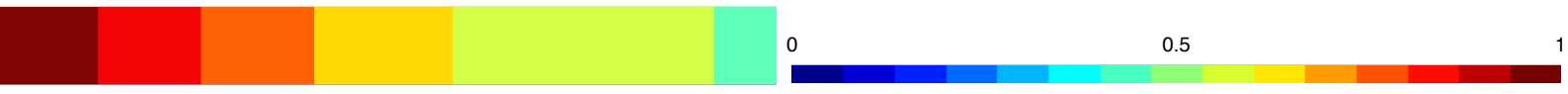

Figure 2: Concentration in the cylindrical pore

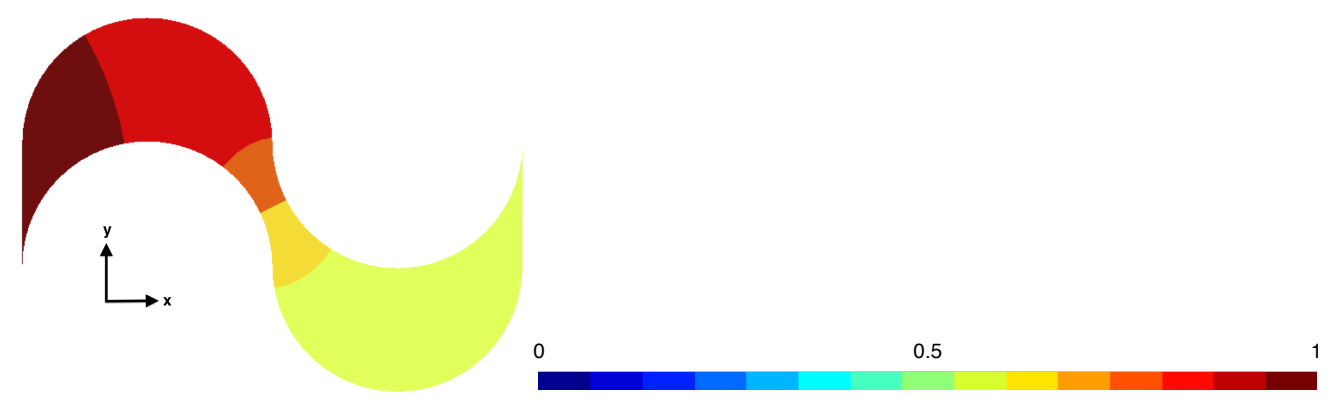

Figure 3: Concentration in a tortuous pore

In conclusion, the tortuous pore may be represented by means of a straight pore of equal volume, orientated in the direction of the net mass flux, and of length equal to the length of the tortuous pore projected onto the direction of the net mass flux. The latter will be referred to as the equivalent pore. The mass flux in the tortuous pore can then be expressed as the sum of the diffusive flux in the equivalent pore and a term in the form of a surface integral which accounts for the geometry of the tortuous pore, as stated in equation 55. 


\subsubsection{The surface integrals in the REV}

In the REV, the porous network can be significantly more complex than the previously defined tortuous pore. An equivalent pore of length $L$ and volume $\phi_{l}$ can be constructed, oriented in the direction of the net mass flux. The mass flux averaged over the equivalent pore is then equal to the mass flux averaged over that part of the REV which contains pore water:

$$
\left\langle\boldsymbol{J}_{D}^{i}\right\rangle_{\hat{\Omega}_{e q}}=-D_{i}\left(\nabla\left\langle u_{i}\right\rangle_{\hat{\Omega}_{l}}+\frac{1}{\hat{\Omega}_{l}} \int_{\hat{A}_{l j}} \tilde{u}_{i} \boldsymbol{n}_{l j} d A\right)
$$

where $\hat{\Omega}_{e q}$ refers to the volume of the equivalent pore.

Note that the average of the mass flux in the equivalent pore, as defined in equation 58, accounts for the tortuosityconnectivity of that part of the elementary volume which contains pore water. The last term reduces the mass flux in the direction parallel to the equivalent pore. Furthermore, the direction of the equivalent pore is parallel to the direction of $\nabla\left\langle u_{i}\right\rangle_{\Omega_{l}}$. Taking this into account, equation 58 can be approximated as:

$$
\begin{aligned}
\left\langle\boldsymbol{J}_{D}^{i}\right\rangle_{\hat{\Omega}_{e q}} & \simeq-D_{i}\left(\nabla\left\langle u_{i}\right\rangle_{\hat{\Omega}_{l}}-\sigma \nabla\left\langle u_{i}\right\rangle_{\hat{\Omega}_{l}}\right) \\
& =-D_{i}\left([1-\sigma) \nabla\left\langle u_{i}\right\rangle_{\hat{\Omega}_{l}}\right)
\end{aligned}
$$

where $\sigma$ is a dimensionless positive function which accounts for the reduction of the mass flux in the direction of the equivalent pore.

Finally, the average of the mass flux in the REV is obtained by multiplying equation 59 by $\phi_{l}$ :

$$
\left\langle J_{D}^{i}\right\rangle_{\hat{\Omega}}=-\phi_{l} D_{i}[1-\sigma] \nabla\left\langle u_{i}\right\rangle_{\hat{\Omega}_{l}}
$$

\subsubsection{The final transport equation in the equivalent pore}

Based on the results obtained in section 4.6.2, the transport equation in the equivalent pore related to the REV is given by:

$$
\begin{aligned}
& \frac{\partial}{\partial t}\left(\phi_{l}\left\langle u_{i}\right\rangle_{\hat{\Omega}_{l}}\right)+\nabla \cdot\left(\left\langle u_{i}\right\rangle_{\hat{\Omega}_{l}} \frac{k_{l}}{v_{l}}[1-\sigma] \nabla\left\langle p_{c}\right\rangle_{\hat{\Omega}_{l}}\right) \\
& =\nabla \cdot\left(\phi_{l} D_{i}[1-\sigma] \nabla\left\langle u_{i}\right\rangle_{\hat{\Omega}_{l}}\right) \\
& +\frac{z_{i} F}{R} \nabla \cdot\left(\phi_{l} D_{i} T^{-1}\left\langle u_{i}\right\rangle_{\hat{\Omega}_{l}}[1-\sigma] \nabla\langle\Phi\rangle_{\hat{\Omega}_{l}}\right) \\
& +\nabla \cdot\left(\phi_{l} D_{i}\left\langle u_{i}\right\rangle_{\hat{\Omega}_{l}}[1-\sigma] \nabla\left\langle\ln \gamma_{i}\right\rangle_{\hat{\Omega}_{l}}\right) \\
& +\nabla \cdot\left(\phi_{l} D_{i}\left\langle u_{i}\right\rangle_{\hat{\Omega}_{l}}\left\langle\ln a_{i}\right\rangle_{\hat{\Omega}_{l}}[1-\sigma] \nabla\langle\ln T\rangle_{\hat{\Omega}_{l}}\right) \\
& +\phi_{l}\left\langle r_{i}\right\rangle_{\hat{\Omega}_{l}}
\end{aligned}
$$

Finally, defining the tortuosity-connectivity parameter as $\tau=1-\sigma$, the final transport equation is obtained:

$$
\begin{aligned}
& \frac{\partial}{\partial t}\left(\phi_{l}\left\langle u_{i}\right\rangle_{\hat{\Omega}_{l}}\right)+\nabla \cdot\left(\left\langle u_{i}\right\rangle_{\hat{\Omega}_{l}} \frac{k_{l}}{v_{l}} \tau \nabla\left\langle p_{c}\right\rangle_{\hat{\Omega}_{l}}\right) \\
& =\nabla \cdot\left(\phi_{l} D_{i} \tau \nabla\left\langle u_{i}\right\rangle_{\hat{\Omega}_{l}}\right) \\
& +\frac{z_{i} F}{R} \nabla \cdot\left(\phi_{l} D_{i} T^{-1}\left\langle u_{i}\right\rangle_{\hat{\Omega}_{l}} \tau \nabla\langle\Phi\rangle_{\hat{\Omega}_{l}}\right) \\
& +\nabla \cdot\left(\phi_{l} D_{i}\left\langle u_{i}\right\rangle_{\hat{\Omega}_{l}} \tau \nabla\left\langle\ln \gamma_{i}\right\rangle_{\hat{\Omega}_{l}}\right) \\
& +\nabla \cdot\left(\phi_{l} D_{i}\left\langle u_{i}\right\rangle_{\hat{\Omega}_{l}}\left\langle\ln a_{i}\right\rangle_{\hat{\Omega}_{l}} \tau \nabla\langle\ln T\rangle_{\hat{\Omega}_{l}}\right) \\
& +\phi_{l}\left\langle r_{i}\right\rangle_{\hat{\Omega}_{l}}
\end{aligned}
$$




\section{Determining the tortuosity-connectivity parameter}

\subsection{Darcy versus Hagen-Poiseuille flows}

The tortuosity-connectivity parameter can be determined by comparing the Darcy flux with the Hagen-Poiseuille fluxes in a cylindrical pore. The following analysis, it should be noted, is valid only for homogeneous materials. Consider a homogeneous material, partially saturated with pore water which is assumed to be continuously and uniformly distributed. Then the equivalent pore related to the porous network which contains pore water can be chosen to be a cylindrical pore of constant section. In that case, the Darcy and Hagen-Poiseuille fluxes must coincide, which leads to the expression of the tortuosity-connectivity as a function of the permeability of the porous medium.

Based on the obtained results, the Darcy flux in the equivalent pore can be expressed as:

$$
\left\langle J_{\text {Darcy }}^{i}\right\rangle_{\hat{\Omega}}=-\frac{\hat{k}_{l}}{v_{l}} \nabla\left\langle p_{l}\right\rangle_{\hat{\Omega}}
$$

where $\hat{k}_{l}=\tau k_{l}$ is now a macroscopic function which describes the permeability of the complex and tortuous porous network.

The Hagen-Poiseuille flux in the equivalent pore reads:

$$
\left\langle\boldsymbol{J}_{\boldsymbol{H}-\boldsymbol{P}}^{i}\right\rangle_{\hat{\Omega}}=-\tau \frac{r^{2}}{8 v_{l}} \nabla\left\langle p_{l}\right\rangle_{\hat{\Omega}}
$$

where $r$ is the radius of the pore.

Setting equations 63 and 64 equal, the tortuosity-connectivity parameter can be written as a function of the permeability function:

$$
\tau(r)=\frac{8 \hat{k}_{l}}{r^{2}}
$$

The tortuosity-connectivity for a fully saturated homogeneous material can be calculated from equation 65 with $r=R$, where $R$ is the radius of the saturated equivalent pore associated to a fully saturated REV:

$$
\tau(R)=\frac{8 \hat{k}_{l}}{R^{2}}
$$

The radii $r$ and $R$ are related according to the following expression:

$$
r^{2}=R^{2} \frac{\phi_{l}}{\phi}
$$

where $\phi$ is the porosity of the REV.

The tortuosity parameter can now be expressed as a function of the pore water content:

$$
\tau\left(\phi_{l}\right)=\frac{8 \hat{k}_{l}}{R^{2}} \frac{\phi}{\phi_{l}}
$$

The permeability is often expressed as the product of an intrinsic permeability coefficient $\hat{K}\left(m^{2}\right)$ and a permeability function relative to the $l$-phase $\hat{k}_{r l}$, which takes values between 0 and 1 . Equation 68 can then finally be rewritten as:

$$
\tau\left(\phi_{l}\right)=\tau(\phi) \frac{\phi}{\phi_{l}} \hat{k}_{r l}\left(\phi_{l}\right)
$$




\subsection{Calculation of the tortuosity-connectivity parameter}

Experimental measurements of the tortuosity-connectivity would require determining the ttortuosity-connectivity for various values of the pore water content, which should be distributed uniformly within the material. Methods to obtain a uniformly distributed pore water content in concrete can be found in research work $(24,25,26)$.

According to equation 69, the tortuosity-connectivity parameter of the porous medium can be calculated if its permeability is known. The permeability is often modelled by means of the van Genuchten model:

$$
\hat{k}_{l}\left(\phi_{l}\right)=\hat{K} \sqrt{\frac{\phi_{l}}{\phi}}\left(1-\left[1-\left(\frac{\phi_{l}}{\phi}\right)^{\frac{1}{e}}\right]^{e}\right)
$$

where $e$ is a material constant.

The expression for the tortuosity-connectivity parameter becomes then:

$$
\tau\left(\phi_{l}\right)=\tau(\phi) \sqrt{\frac{\phi}{\phi_{l}}}\left(1-\left[1-\left(\frac{\phi_{l}}{\phi}\right)^{\frac{1}{e}}\right]^{e}\right)
$$

In order to calibrate this model with experimental results, Equation 711 was used in this study, where the material constant $e$ and the tortuosity-connectivity in saturated conditions $\tau(\phi)$ were measured for four different concretes, by fitting Darcy's law to experimentally obtained data.

The four concretes were designed in acordance with usual mixes used in agressive environments to improve the impermeability and resistance against penetration of ionic species. Concrete dosages are referred to as mat 1 , mat2, mat 3 and mat. The cement type used for materials mat1 (without additions), mat2 (with addition of $20 \%$ of fly ash) and mat 4 (with addition of of $10 \%$ of silica fume) is I $42.5 \mathrm{R} / \mathrm{SR}$ according to the RC-16 Standard (?). The cement employed for material mat 3 contains $66 \%$ blast furnace slag and its type is known as III/B $42.5 \mathrm{~L} / \mathrm{SR}$, in agreement with the RC-16 Standard (? ). The properties of the materials are described in detail in (28). The dosages of the materials are given in table 1.

\begin{tabular}{lrrrr}
\hline \multicolumn{5}{c}{ Dosages $\left(\mathrm{kg} / \mathrm{m}^{3}\right)$} \\
\hline Material & mat1 & mat2 & mat3 & mat4 \\
\hline Cement & 380 & 357 & 380 & 304 \\
Water & 171 & 194 & 171 & 154 \\
Fly ash & 0 & 76 & 0 & 0 \\
Silica fume & 0 & 0 & 0 & 38 \\
Aggregate & 787 & 770 & 787 & 800 \\
Sand & 1022 & 966 & 1022 & 1067 \\
Superplasticizer* & 0.97 & 0.70 & 1.30 & 1.80 \\
\hline * of cement weight & & & &
\end{tabular}

Table 1: Dosages of the studied materials

The tortuosity-connectivity function of the materials, obtained from the experimental results, is plotted in figure 4. It is shown that the tortuosity-connectivity parameter decreases significantly for lower pore water contents, meaning that the mass fluxes are significantly reduced in the direction of the net mass flux. In saturated conditions, the tortuosity-connectivity functions reach their maxima. It can be observed that the tortuosity-connectivity undergoes hysteresis, which is due to the fact that the pore water can get trapped in ink-bottle shaped pores when the concrete is subjected to drying conditions (5). It is worth noting that this phenomenon has been found significantly greater for mat 1 and mat2, probably due to a significantly higher amount of bottleneck pores, being the two mixtures with the higher total porosity as can be seen in (28). Similarly, mat1 and mat2 presented the greater value of the 
tortuosity-connectivity parameter. In the case of mat3 and mat4, the tortuosity-connectivity parameter obtained was considerably lower, with also a lower total porosity and average pore size (28) than the rest. This is coherent with the results obtained by (28) for differential thermal analysis, mercury intrusion porosimetry, water and gas per-meability and mechanical properties of The materials, in which the mixtures could be divided into two groups according to its similar behaviour, presenting better performance for mixtures mat 3 and mat4.

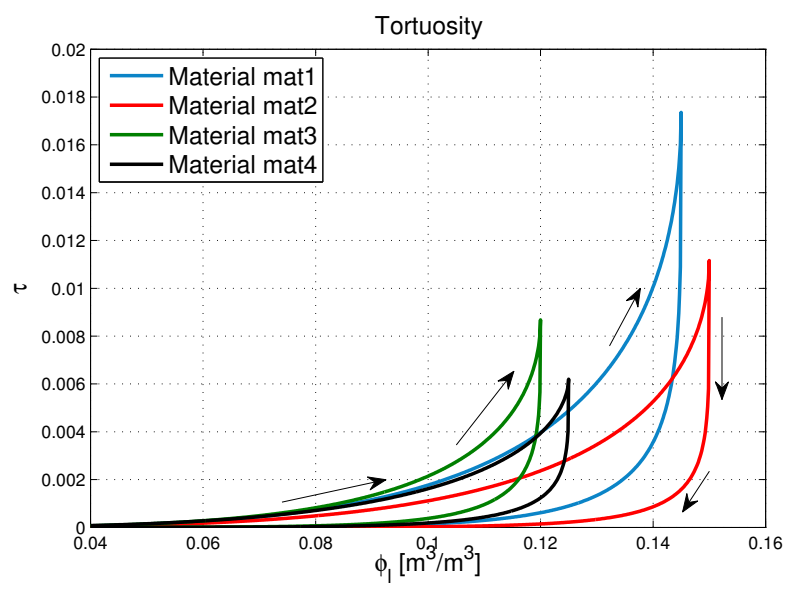

Figure 4: The tortuosity as a function of the pore water content

Using these tortuosity-connectivity functions, the diffusion coefficient for ionic species $\tau\left(\phi_{l}\right) \phi_{l} D_{i}$ can be obtained. Such coefficients were measured for chloride ions and for several pore water contents. As can be deduced from figure 4 , the diffusion coefficient $\tau\left(\phi_{l}\right) \phi_{l} D_{i}$ increases with the pore water content.

\subsection{Closure equations}

As shown in (5), the terms in equation 62 related to migration and chemical activity can generally be ignored. For the sake of simplicity, the same assumptions are made in this paper, yielding:

$$
\begin{aligned}
\frac{\partial}{\partial t}\left(\phi_{l}\left\langle u_{i}\right\rangle_{\hat{\Omega}_{l}}\right) & +\nabla \cdot\left(\left\langle u_{i}\right\rangle_{\hat{\Omega}_{l}} \frac{k_{l}}{v_{l}} \tau \nabla\left\langle p_{c}\right\rangle_{\hat{\Omega}_{l}}\right) \\
& =\nabla \cdot\left(\phi_{l} D_{i} \tau \nabla\left\langle u_{i}\right\rangle_{\hat{\Omega}_{l}}\right)+\phi_{l}\left\langle r_{i}\right\rangle_{\hat{\Omega}_{l}}
\end{aligned}
$$

The capillary pressure $\left\langle p_{c}\right\rangle_{\hat{\Omega}_{l}}$ was modelled by means of the capillary model proposed in (5):

$$
\left\langle p_{c}\right\rangle_{\hat{\Omega}_{l}}=A\left(\operatorname{atan}\left(a\left[b-\phi_{l}\right]\right)+\operatorname{atan}(a[\phi-b])\right)
$$

where $A(M P a)$ is the capillary modulus, and $a$ and $b$ are material constants.

The reaction term $\left\langle r_{i}\right\rangle_{\hat{\Omega}_{l}}$ was modelled by means of the Langmuir adsorption equation, which in this case can be expressed as (5):

$$
\phi_{l}\left\langle r_{i}\right\rangle_{\hat{\Omega}_{l}}=\phi_{l}\left\langle\hat{u}_{i}\right\rangle_{\hat{\Omega}_{l}} \hat{\Omega}_{l}^{-1} k_{e q} \frac{\partial}{\partial t}\left(\frac{\phi_{l}\left\langle u_{i}\right\rangle_{\hat{\Omega}_{l}}}{\hat{\Omega}_{l}+k_{e q} \phi_{l}\left\langle u_{i}\right\rangle_{\hat{\Omega}_{l}}}\right)
$$

where $k_{e q}\left(\mathrm{~m}^{3} / \mathrm{kg}\right)$ is a constant which describes the equilibrium between the free ions and the bound ions, while $\left\langle\hat{u}_{i}\right\rangle_{\hat{\Omega}_{l}} \hat{\Omega}_{l}^{-1}\left(\mathrm{~kg} / \mathrm{m}^{3}\right)$ is the maximum density of reactants in volume $\hat{\Omega}_{l}$.

Substituting equations $70,71,73$ and 74 into equation 72 , the closure equations are obtained. The closure equation for chloride ions was solved by means of the finite element method. Some results are shown in the next section. 


\section{Numerical simulations of experimental chloride profiles}

\subsection{Overview of the experimental programme}

Once the tortuosity-connectivity parameter had been calibrated with concretes widely studied by the authors and its good fit had been verified to adequately simulate the experimental results obtained, the model was tested by comparing the numerical simulations with experimental chloride profiles obtained from two different concretes. The latter were obtained from two different mixtures (referred to as mat5 and mat6) selected to represent the two types of behaviour observed in the previous experimental campaign. This way, in the new experimental campaign the reference concrete mat 5 is a mixture without additions representing the group mat 1 and mat 2 , and mat 6 is a mixture containing $10 \%$ of silica fume, representing the group with the best general durable perfomance mat 3 and mat4. Both dosages were based on Portland cement. The water-cementitious materials ratio were 0.40 and 0.45 for the concrete with silica fume. More details are given in table 2. These materials were tested under several boundary conditions in order to experimentally simulate half a year of service life of a concrete subjected to high mountain environment with melting salts, which is a highly aggresive environment with chlorides. The initially fully saturated concrete samples were subjected to five different aggressive environments for 180 days. The first 75 days, the samples were exposed to a chloride solution of $100 \mathrm{~g} / \mathrm{l}$ at a temperature of $1^{\circ} \mathrm{C}$. This phase simulates the winter season in which chloride solutions are sprayed over the roads and concrete structures in order to avoid the forming of ice patches on the road surface. As a result, the chloride ions start to penetrate into the concrete. On day 76, a thin layer $(2 \mathrm{~cm})$ of fresh water was poured onto the exposed surface at a temperature of $10^{\circ} \mathrm{C}$, and was removed 30 days after. This phase corresponds to a rainy spring. The rain eliminates chlorides from the concrete structures. In order to simulate a drier summer, for the next 60 days, the concrete was dried at a relative humidity of $75 \%$ and temperatures of $T=16^{\circ} \mathrm{C}$ and $T=5^{\circ} \mathrm{C}$ (30 days each). During that season, the chloride ions are transported towards the surface, where they start to precipitate. Finally, the samples were exposed once more to a chloride solution of $100 \mathrm{~g} / \mathrm{l}$ at temperature $3^{\circ} \mathrm{C}$ for 15 days. A schematic representation is given in table 3 .

\begin{tabular}{lrr}
\hline \multicolumn{3}{c}{ Dosages $\left(\mathrm{kg} / \mathrm{m}^{3}\right)$} \\
\hline Material & mat5 & mat6 \\
\hline Cement & 400 & 320 \\
Water & 160 & 180 \\
Silica fume & 0 & 40 \\
Ratio water/cementitious materials & 0.40 & 0.45 \\
Aggregate & 970 & 1015.73 \\
Sand & 846 & 861.08 \\
Superplasticizer* & 1.5 & 1.5 \\
\hline *\% of cement weight & &
\end{tabular}

Table 2: Dosages of the studied materials.

\begin{tabular}{llll}
\hline \multicolumn{4}{c}{ Aggressive environments } \\
\hline & Chloride con- & Relative \\
centration $(g / l)$ & Temperature \\
hays & cemity $(\%)$ & $\left({ }^{\circ} C\right)$ \\
\hline $1-75$ & 100 & 100 & 1 \\
$76-105$ & 0 (at day 76$)$ & 100 & 10 \\
$106-135$ & - & 75 & 16 \\
$136-165$ & - & 75 & 5 \\
$166-180$ & 100 & 100 & 3 \\
\hline
\end{tabular}

Table 3: The aggressive environments 


\subsection{Numerical modelling}

In order to account for the relative humidity and temperature, the transport model was coupled to differential equations based on Darcy's law (to solve for the pore water content) and on Fourrier's law (to solve for temperature). The coupled differential equations were solved by means of the finite element method. A detailed description of the formulation can be found in (5). The model was then calibrated for the materials as described in (5).

The numerical results versus the experimental chloride profiles are plotted in figures 5-7 for material mat5 and figures 8-10 for material mat6. The vertical axis represents the chloride profiles expressed in $\mathrm{kg} / \mathrm{m}^{3}$ of concrete, while the horizontal axis represents the penetration depth expressed in $\mathrm{mm}$. It can be observed that a good fit is obtained as numerical simulations reproduce the experimental results with a significantly good fit. The capillary model enabled modelling of the pore water flow, accounting for the properties of the microstructure of the material. The microstructure was characterized by the tortuosity-connectivity parameter which was quantified in function of the pore radii for different materials.
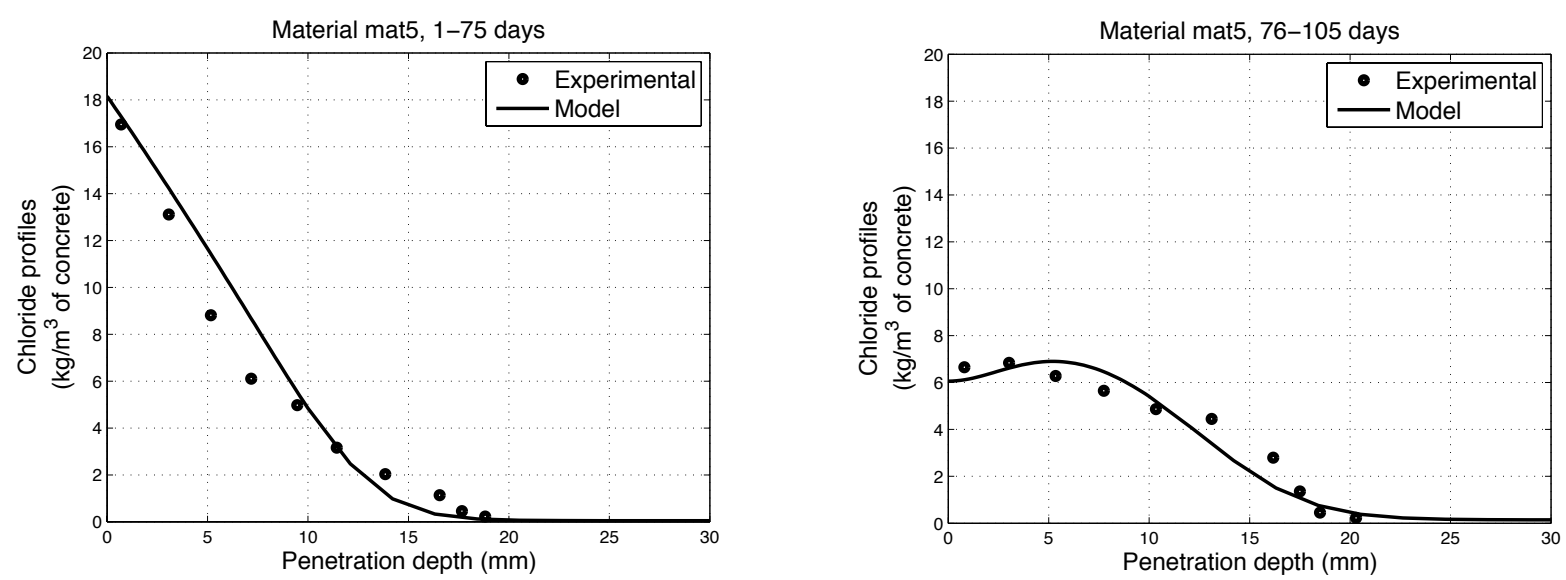

Figure 5: Experimental versus numerical solution for material mat5 and environments 1 (1-75 days) and 2 (76-105 days)
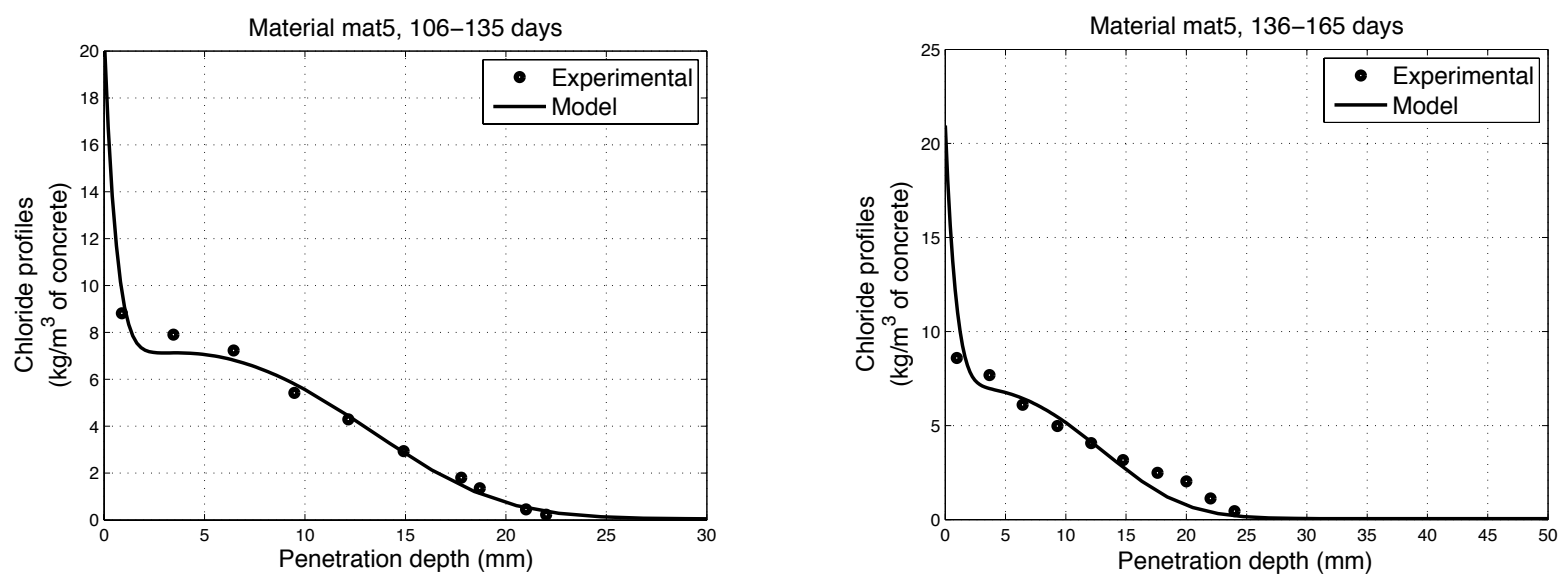

Figure 6: Experimental versus numerical solution for material mat5 and environments 3 (106-135 days) and 4 (136-165 days) 

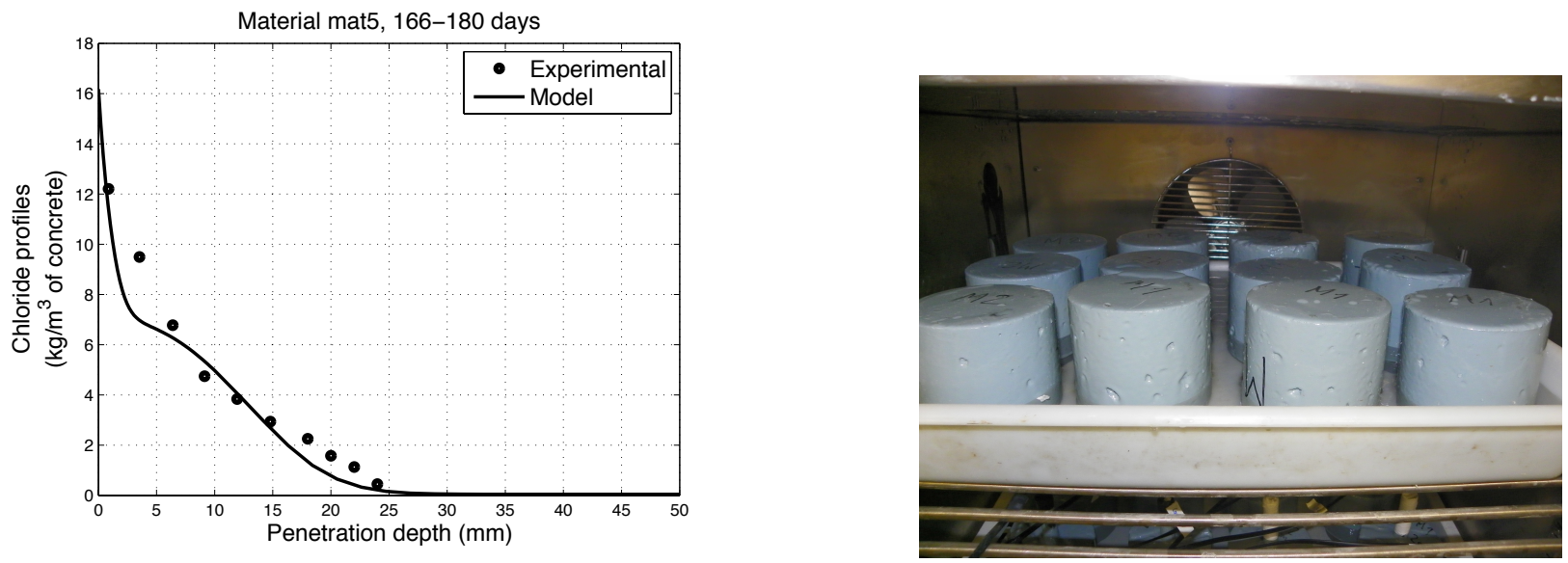

Figure 7: Left: experimental versus numerical solution for material mat 5 and environment 5 (166-180 days). Right: concrete samples exposed to water
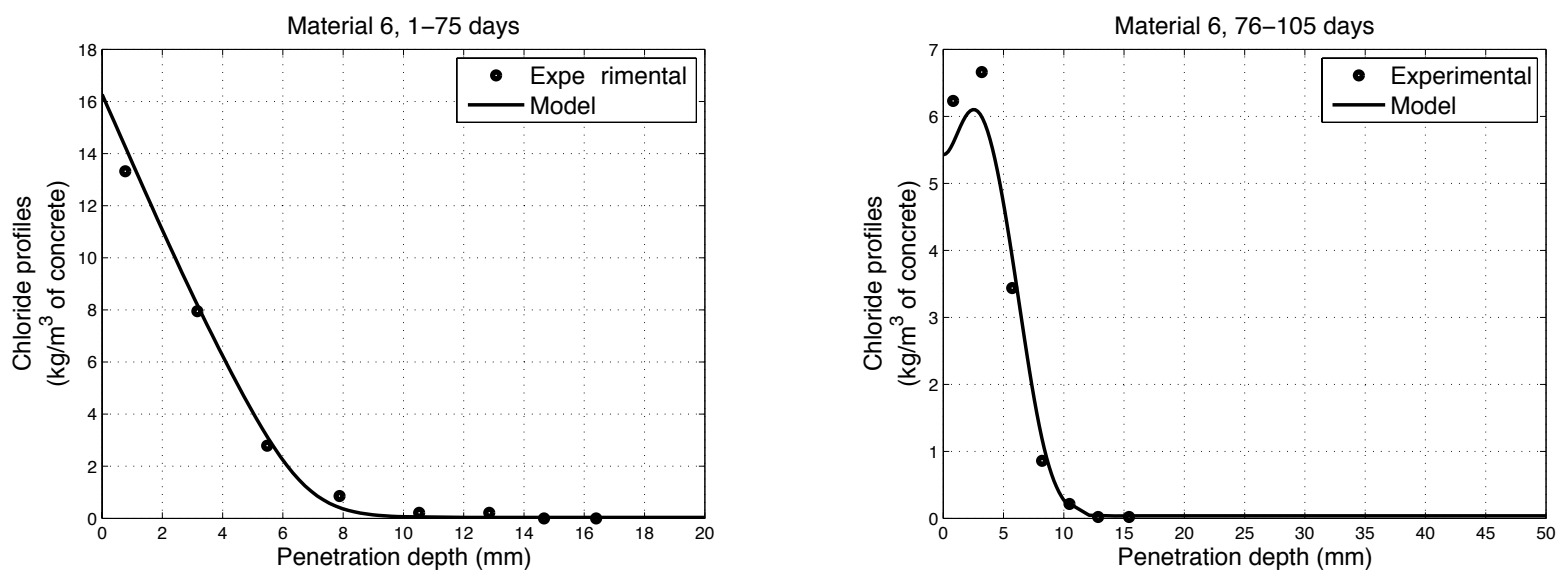

Figure 8: Experimental versus numerical solution for material mat6 and environments 1 (1-75 days) 2 (76-105 days)
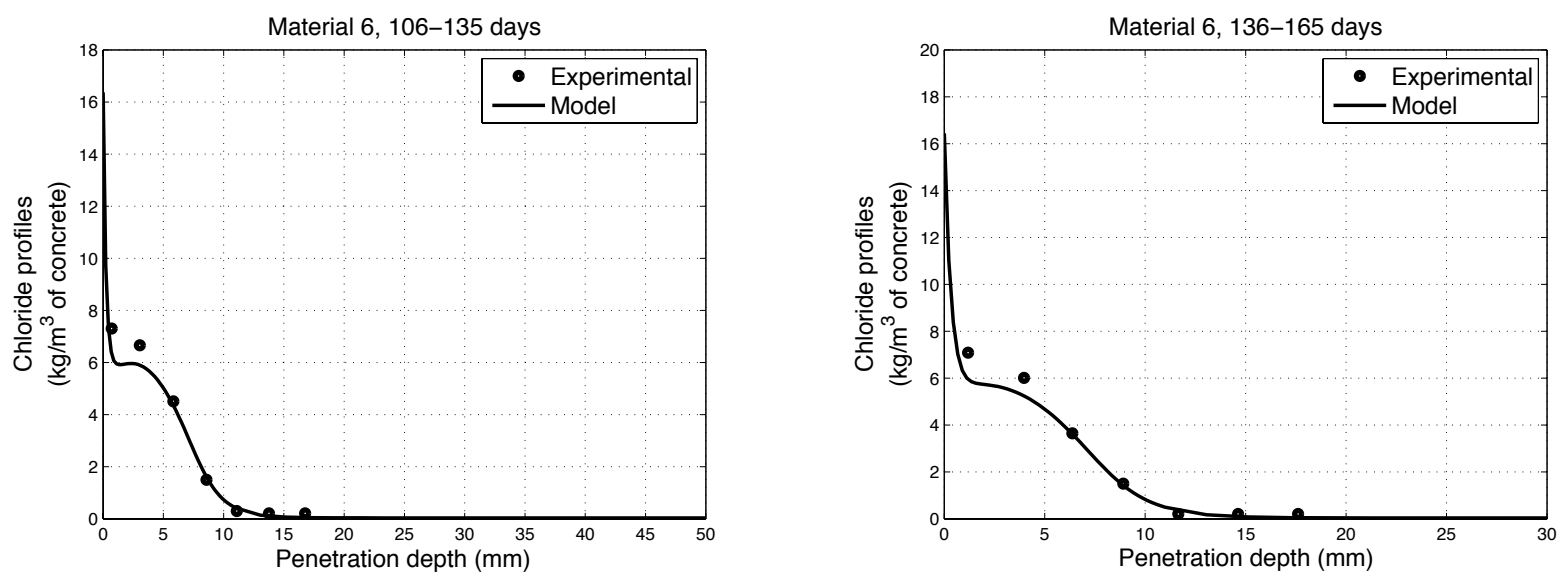

Figure 9: Experimental versus numerical solution for material mat6 and environments 3 (106-135 days) and 4 (136-165 days) 

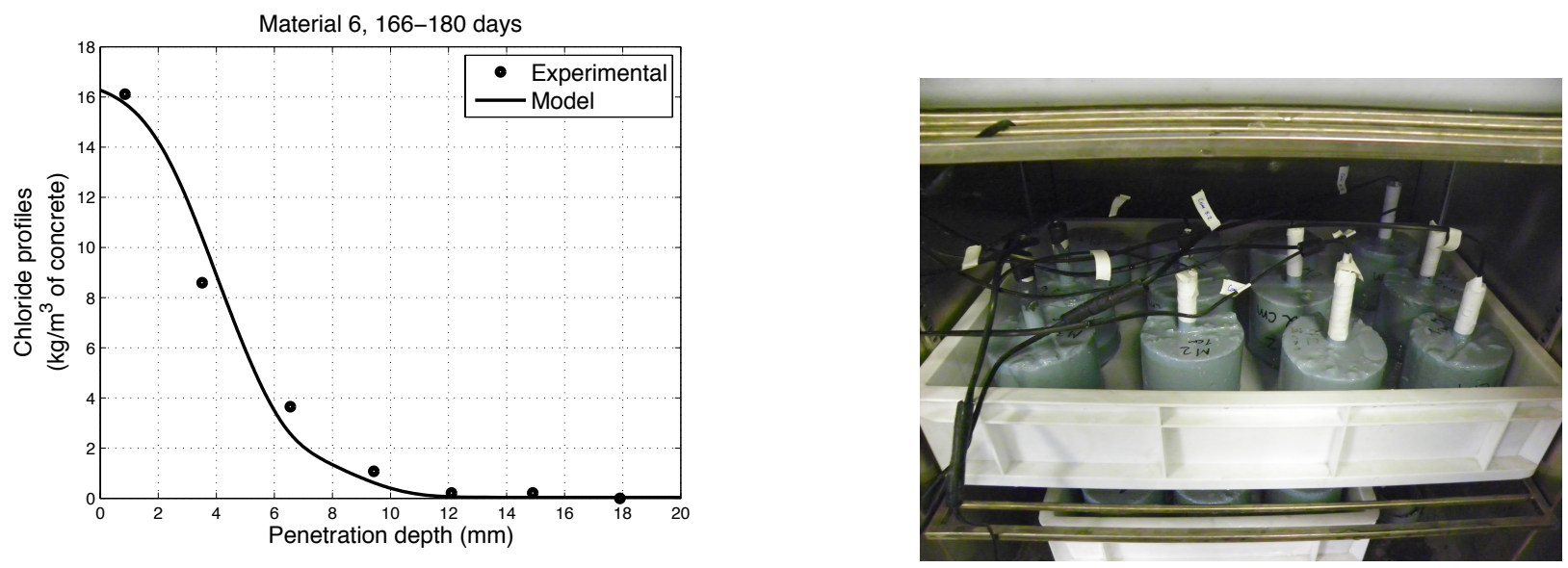

Figure 10: Left: experimental versus numerical solution for material mat6 and environment 5 (166-180 days). Right: measurements of the relative humidity within the concrete samples 


\section{Conclusions}

The study carried out in this work led to the following conclusions:

1. In order to obtain the transport equations at the macroscopic scale, the microscopic equations were integrated over the REV by means of the well known averaging technique.

2. It was shown that the dispersion terms which arose from the averaging procedure can be ignored.

3. The surface integrals which arose from the averaging technique were interpreted. It was shown not only how they are related to the tortuosity-connectivity of the porous network, but also how they influence the mass fluxes of the ionic species.

4. The transport equations in the REV were compared with the equations in an equivalent pore, oriented in the direction of the net mass flux.

5. An explicit expression of the ttortuosity-connectivity parameter was obtained by comparing Darcy's law and the Hagen-Poiseuille equations for the equivalent pore.

6. The proposed model accurately fits the results obtained from experimental tests performed on concretes in non-saturated conditions.

\section{Acknowledgements}

The authors gratefully acknowledge the financial support provided by Ministry of Economy and Competitiveness of Spain by means of the Research Fund Project PID2019-108978RB-C31.

\section{References}

[1] Ministry of the Presidency, Madrid, Spain, EHE - Code on Structural Concrete (August 2008)

[2] S. Guzmán, J. Gálvez, J. Sancho, Cover cracking of reinforced concrete due to rebar corrosion induced by chloride penetration, Cement and Concrete Research 41 (8) (2011) 893 - 902.

[3] S. Guzmán, J. C. Gálvez, J. M. Sancho, Modelling of corrosion-induced cover cracking in reinforced concrete by an embedded cohesive crack finite element, Engineering Fracture Mechanics 93 (0) (2012) 92 - 107.

[4] L. Bertolini, B. Elsener, P. Pedeferri, R. Polder, Corrosion of steel in concrete, WILEY-VCH Verlag GmbH \& Co. KGaA.

[5] M. Fenaux, Modelling of chloride transport in non-saturated concrete. From Microscale to Macroscale, Ph.D. thesis, Escuela Técnica Superior de Ingenieros de Caminos, Canales y Puertos, Madrid, Spain (May 2013).

[6] E. Samson, J. Marchand, Numerical Solution of the Extended Nernst-Planck Model, Journal of Colloid and Interface Science 215 (1) (1999) $1-8$.

[7] E. Samson, J. Marchand, Modeling the effect of temperature on ionic transport in cementitious materials, Cement and Concrete Research 37 (3) (2007) $455-468$.

[8] O. Coussy, F. Ulm, Elements of durability mechanics of concrete structures, in: F. Ulm, Z. Bažant, F. Wittmann (Eds.), Creep, Shrinkage and Durability Mechanics of Concrete and other Quasi-Brittle Materials, Elsevier, Oxford, United Kingdom, 2001, pp. $393-409$.

[9] J. Marchand, B. Gérard, A. Delagrave, Ion transport mechanisms in cement-based materials, in: Materials Science of Concrete, Vol. 5, American Ceramic Society, 1998, pp. 307-400.

[10] J. Marchand, Y. Maltais, E. Samson, V. Johansen, K. Hazrati, Modeling interaction mechanisms in cement-based materials - An overview, in: Sidney Diamond Symposium on Materials Science of Concrete and Cementitious-based Composites, American Ceramic Society, 1998, pp. 143-162.

[11] C. Page, N. Short, A. E. Tarras, Diffusion of chloride ions in hardened cement pastes, Cement and Concrete Research 11 (3) (1981) 395 406.

[12] S. Goto, D. Roy, Diffusion of ions through hardened cement pastes, Cement and Concrete Research 11 (5-6) (1981) $751-757$.

[13] A. Saetta, R. Scotta, R. Vitaliani, Analysis of Chloride Diffusion into Partially Saturated Concrete, Materials Journal 90 (5) (1993) 441 - 451.

[14] B. Martín-Pérez, H. Zibara, R. Hooton, M. Thomas, A study of the effect of chloride binding on service life predictions, Cement and Concrete Research 30 (8) (2000) $1215-1223$.

[15] S. Ahmad, A. Azad, K. Loughlin, Effect of the key mixture parameters on tortuosity and permeability of concrete, Journal of Advanced Concrete Technology 10 (3) (2012) 86-94.

[16] M. S. Ray, Diffusion in zeolites and other microporous solids, by j. karger and d. m. ruthven, john wiley, new york, usa (1992). 605 pages. isbn 0-47 1-50907-8., Developments in Chemical Engineering and Mineral Processing 4 (3-4) (1996) $254-254$.

[17] F. Dullien, Porous Media, Fluid Transport and Pore Structure, Academic Press, New York, USA, 1979.

[18] C. Andrade, R. D'Andrea, N. Rebolledo, Calculation of tortuosity factor for the model based in concrete resistivity.

[19] W. G. Gray, A derivation of the equations for multi-phase transport, Chemical Engineering Science 30 (2) (1975) 229 - 233.

[20] F. A. Howes, S. Whitaker, The spatial averaging theorem revisited, Chemical Engineering Science 40 (8) (1985) 1387 - 1392. 
[21] Y. Bachmat, J. Bear, Macroscopic modelling of transport phenomena in porous media. 1: The continuum approach, Transport in Porous Media 1 (3) (1986) 213-240.

[22] C. Soulaine, G. Debenest, M. Quintard, Upscaling multi-component two-phase flow in porous media with partitioning coefficient, Chemical Engineering Science 66 (23) (2011) 6180 - 6192.

[23] M. Mainguy, O. Coussy, V. Baroghel-Bouny, Role of Air Pressure in Drying of Weakly Permeable Materials, Journal of Engineering Mechanics 127 (6) (2001) 582-592.

[24] A. Guimarães, M. Climent, G. de Vera, F. Vicente, F. Rodrigues, C. Andrade, Determination of chloride diffusivity through partially saturated portland cement concrete by a simplified procedure, Construction and Building Materials 25 (2) (2011) $785-790$.

[25] M. A. Climent, G. de Vera, J. F. López, E. Viqueira, C. Andrade, A test method for measuring chloride diffusion coefficients through nonsaturated concrete: Part i. the instantaneous plane source diffusion case, Cement and Concrete Research 32 (7) (2002) 1113 - 1123.

[26] G. de Vera, M. A. Climent, E. Viqueira, C. Antón, C. Andrade, A test method for measuring chloride diffusion coefficients through partially saturated concrete. part ii: The instantaneous plane source diffusion case with chloride binding consideration, Cement and Concrete Research 37 (5) (2007) $714-724$.

[27] UNE-EN ISO 12571, Prestaciones higrotérmicas de los productos y materiales para edificios, AENOR, 2000.

[28] S. Mahmoud Abdelkader, E. Reyes Pozo, A. Moragues Terrades, Evolution of microstructure and mechanical behavior of concretes utilized in marine environments, Materials \& Design 31 (7) (2010) 3412 - 3418. 